\title{
Supervisory Control Interface Design for Unmanned Aerial Vehicles through GEDIS-UAV
}

\author{
Salvador Lorite ${ }^{1}$, Adolfo Muñoz ${ }^{1}$, Josep Tornero ${ }^{1}$, Pere Ponsa ${ }^{2}$, and Enric Pastor $^{3}$ \\ ${ }^{1}$ Design and Manufacturing Institute (IDF), Polytechnic University of Valencia, \\ CPI, Edif. 8E, Camino de Vera s/n, 46022 Valencia, Spain \\ \{slorite, amunyoz, jtornero\} aidf.upv.es \\ ${ }^{2}$ Automatic Control Department, UPC BarcelonaTech University, \\ Av. Víctor Balaguer s/n, 08800 Vilanova I la Geltrú, Barcelona, Spain \\ pedro.ponsa@upc.edu \\ ${ }^{3}$ ICARUS Research Group, UPC BarcelonaTech University, \\ C/ Esteve Terradas 7, 08860 Castelldefels, Barcelona, Spain \\ enric@ac.upc.edu
}

\begin{abstract}
This paper reflects the state of art in the field of human factors for unmanned aerial vehicles. It describes the GEDIS-UAV guide, which is a modification of the GEDIS guide. It also shows the evaluation of the Sky-eye project graphical user interface as an example of the methodology. The analysis and evaluation method reflected in this paper may be used to improve the graphical user interface of any unmanned aerial vehicle.
\end{abstract}

Keywords: Supervisory Control, Unmanned Vehicles, Ergonomics, GEDIS.

\section{Introduction}

This paper aims to advance the research on guidelines to design and implement interfaces for monitoring unmanned aerial vehicles (UAVs). Today the UAVs have become a fashionable topic in the world, but we must be aware of the risks associated with a failure of these flying machines, the consequences can be disastrous, even more when these machines are for civilian use. Some failures occur during teleoperation [1], [3], [5], [7], [13], [25]. Twenty percent of the failures are attributed to human error [24]; therefore improving the control interface can decrease the failures considerably [4], [26]. There are not specific regulations or guidelines oriented to the design of interfaces for UAVs, however there is one guideline we consider can be helpful after some adaptations, It is the human factors guide for human supervisory control display design GEDIS [18]. Taking into account aspects of human computer interaction of UAVs, this guide has been adapted in order to make it fully functional for UAV's graphical user interfaces (GUIs), here lays the main contribution of the present work. 


\section{Previous Research on Human Interface Design Guidelines}

This section refers to the state of the art of standards and guidelines related with the design of GUIs for UAVs: ARINC 661 [2], STANAG 4586 [4], [11], DO-178B [20], JAUS [23], ISO 9241-11 [8] and GEDIS [18]. Table 1 shows a comparison of the standards and guidelines.

Table 1. Standards and guidelines comparison

\begin{tabular}{llll}
\hline & User Centered & Focused on the system & Focused on interaction \\
\hline JAUS & NO & YES & NO \\
STANAG & NO & YES & NO \\
ARINC 661 & NO & NO & NO \\
DO-178B & NO & NO & NO \\
ISO 9241 & YES & NO & YES \\
GEDIS & YES & NO & YES \\
\hline
\end{tabular}

To date the unmanned systems architectures vary considerably from one system to another, this situation complicates the creation of a guideline or a standard. There are guidelines and standards that can be used but they present limitations because they do not meet all the needs of each unmanned system architecture, for example GEDIS should be modified in order to be useful for other types of unmanned vehicles or for other types of missions.

\section{GEDIS-UAV Guideline}

From the initial point of view of strategies for effective human-computer interaction applied to supervision tasks in industrial control rooms [12], [21], GEDIS-UAV has adopted GEDIS guideline method to cover all the aspects of the GUI design [18 - 19]. GEDIS-UAV offers design recommendations in the moment of creating the interface and it also offers recommendations of improvement for interfaces already created. The guide is composed by indicators and subindicators. The method consists in analyzing and measuring each indicator in order to obtain a global evaluation index.

\subsection{Indicators List}

The GEDIS-UAV indicators have been defined from concepts extracted of other generic human factors guidelines [18] and the subindicators have been defined from the same sources but taking into account specific ergonomic criteria, like the level of situational awareness in UAVs, here lays the main contribution of the present work. The indicators are: architecture, distribution, navigation, color, text font, status of the devices, process values, graphs and tables, data-entry commands, and alarms. 


\subsection{Evaluation}

The evaluation is expressed with a quantitative numeric form and with a qualitative format that reflects the operator experience using the interface or the analysis criteria of the evaluator. The evaluation method is the same as in GEDIS guide. Each subindicator is punctuated numerically in a scale from 1 to 5 . The indicator value is calculated by solving the following formula:

$$
\text { Indicator }=\frac{\sum_{j=1}^{\mathrm{j}} w_{j} \text { Subind }_{j}}{\sum_{\mathrm{j}=1}^{\mathrm{j}} w_{j}}
$$

Where, $\mathbf{J}=$ number of Subindicators of the indicator, Subind $=$ subindicator assessment value and $\mathrm{w}=$ weight. For this study each subindicator has the same weight ( $w 1$ $=\mathrm{w} 2 \ldots=\mathrm{wJ}=1$ ).

The indicators values are used to calculate the global evaluation by solving the following formula:

$$
\text { Global Index }=\frac{\sum_{\mathrm{i}=1}^{10} p_{i} i \text { ind }_{i}}{\sum_{\mathrm{i}=1}^{10} p_{i}}
$$

Where, ind $=$ indicator and $\mathrm{p}=$ weight. As explained before in this first approach all indicators have the same weight $(\mathrm{p} 1=\mathrm{p} 2 \ldots=\mathrm{p} 10=1)$. The guide recommends that the global evaluation index should not be lower than 3 points. A positive evaluation should reach at least 4 points.

\section{Applying the GEDIS-UAV Guide: The Sky-Eye Project Case}

Sky-eye project is part of the work being conducted by the research group ICARUS (Intelligent communications and avionics for robust unmanned aerial systems) of the UPC BarcelonaTech University. Among the group's work, the project Sky-eye [14] aims to research improvements in unmanned aerial systems (UAS) for fire eradication missions, building flexible and generic missions designed for an efficient execution, one of the objectives in order to facilitate the operation is to achieve the appropriate level of automation [22] over all the UAV's work processes; this goal includes the development of a good supervisory control interface. The embedded hardware/software architecture developed by the ICARUS group includes the GCS [15 - 16]. The GCS has been designed to fulfill the following functions: mission planning, mission control, manual and/or supervised control of the UAV and data manipulation. The design of the console that incorporates the graphical interface of the GCS is based on the standard ARINC 661, comprises a display mounted on a control panel similar to a conventional aircraft cockpit. The GUI consists of a TFT (thin film transistor) screen that displays the flight instruments, the flight controls, the local map, the global map for the control of the mission, the artificial horizon and the navigation camera aboard the vehicle. The local map is in the bottom left of the screen and the global map on the right side just above the engine rpm, fuel and oil pressure gauges. Both the local map and global map can zoom in and out the pictures. 
Figure 1 depicts the GUI developed by the research group ICARUS. Although the design is based on the ARINC 661 standard, the GUI has a high complexity in the sense that almost all the features have been included in one screen. GEDIS-UAV structures all the functionalities in a multi-layer application and it allows the tasks to be distributed between different operators. Starting from this information, it is possible to make an assessment that will lead to identify and propose crucial improvements to the supervisory control interface layout.

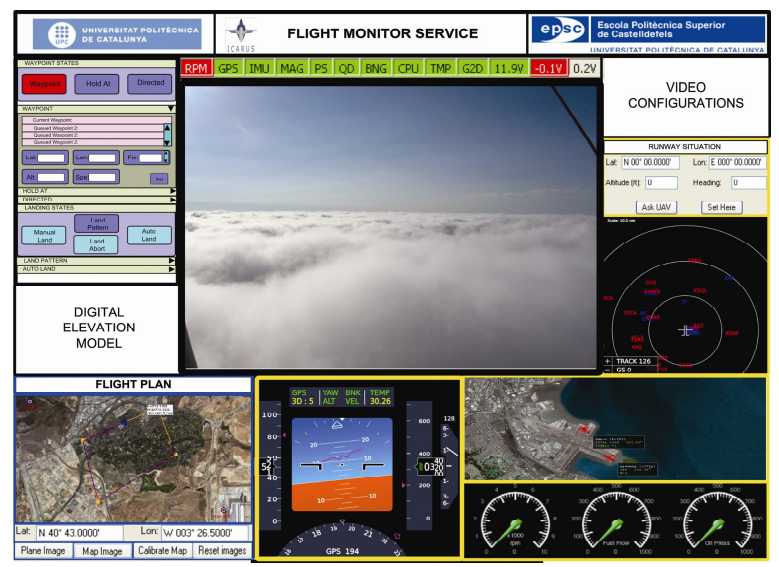

Fig. 1. GUI developed by the research group ICARUS

\section{Current Interface Evaluation through GEDIS-UAV}

The guide detects a group of anomalies and numerically quantifies each of the components and indicators for a global assessment index. As follows the Sky-eye interface end user evaluation (where $\mathrm{A}=$ appropriate, $\mathrm{M}=$ medium, N.A. = Not appropriate):

Table 2. Architecture

\begin{tabular}{lllll}
\hline A: Architecture & A & M & N.A. & Specific criteria \\
\hline A1: Division in areas & 5 & 3 & 0 & --- \\
A2: Screens number "sn" & --- & --- & --- & $3<\mathrm{sn}<9=5, \mathrm{n}<4=0$, \\
\hline
\end{tabular}

The division (A1) got a " 3 " because the mission control module is not appreciable, but it keeps relation with the rest of the modules: UAV, global map, local map, avionics sensors, vehicle control, mission planning and data manipulation. Because there is only one screen that displays all the information together, the number of screens (A2) got a " 0 ", having "1.5" points in total for the architecture indicator (A). Figure $2 \mathrm{~A}$ depicts the original GUI schematic division by areas. 
Table 3. Distribution

\begin{tabular}{lllll}
\hline B: Distribution & A & M & N.A. & Specific criteria \\
\hline B1: Model comparison & 5 & 3 & 0 & --- \\
B2: Flow process & 5 & 3 & 0 & --- \\
B3: Density & 5 & 3 & 0 & --- \\
\hline
\end{tabular}

The model comparison subindicator (B1) got a " 3 " because the interface is similar to the model, except for some items to display. For certain tasks, the process flow is not entirely clear then flow process subindicator (B2) got a "3". All the elements are in close proximity therefore the subindicator density (B3) got a " 0 ", having " 2 " points in total for the distribution indicator (B).

Table 4. Navigation

\begin{tabular}{lllll}
\hline C: Navigation & A & M & N.A. & Specific criteria \\
\hline C1: Navigation between screens & 5 & 3 & 0 & --- \\
\hline
\end{tabular}

The interface has only one screen where all the information is displayed to the operator, this design allows the operator to navigate between different parts of the system but this characteristic limits radically the operator's capability of navigation, one of the main reasons is because every time the operator explores a specific part of the system, another part gets hidden. Another limitation is that the interface does not display the navigation buttons correctly. Therefore the subindicator navigation between screens (C1) got a "0", having " 0 " points in total for the navigation indicator (C).

Table 5. Color

\begin{tabular}{lllll}
\hline D: Color & A & M & N.A. & Specific criteria \\
\hline D1: Absence of non-appropriate & --- & --- & --- & Yes $=5$, No=0 \\
D2: Colors number "cn“ & --- & --- & --- & $\mathrm{cn}<4=5, \mathrm{cn}>4=0$ \\
D3: Blink absence & --- & --- & --- & Yes $=5$, No=0 \\
D4: SC contrast vs GC & 5 & 3 & 0 & --- \\
D5: Relationship with text & 5 & 3 & 0 & --- \\
\hline
\end{tabular}

The absence of non-appropriate combinations subindicator (D1) got a " 5 ". There are more than 9 colors then the subindicator colors number (D2) got a " 0 ". The subindicator (D3) blink absence got a " 5 " because the interface does not have visual alarms. In general there is a good contrast between the graphical contrast and the screen contrast therefore the subindicator (D4) got a " 5 " and the subindicator relationship with text (D5) got a "5" because the relationship with the text color in general is appropriate. All this subindicators lead to "4" points in total for the color indicator (D). 
Table 6. Text font

\begin{tabular}{lllll}
\hline E: Text font & A & M & N.A. & Specific criteria \\
\hline E1: Font number "fn" & --- & --- & --- & fn $<4=5, \mathrm{fn}>4=0$ \\
E2: Absence of small fonts & --- & --- & --- & Yes $=5, \mathrm{No}=0$ \\
E3: Absence of N.A. combinations & --- & --- & --- & Yes $=5, \mathrm{No}=0$ \\
E4: Abbreviation use & 5 & 3 & 0 & --- \\
\hline
\end{tabular}

The number of fonts used in the interface is 3 then the subindicator font number (E1) got a "5". There are some fonts with size 6; therefore the subindicator (E2) got a "0". There are not non-appropriate combinations therefore the subindicator (E3) got a " 5 ", and since the interface uses too many abbreviations the subindicator (E4) got a " 0 ", having " 2.5 " points in total for the text font indicator (E).

Table 7. Status and devices

\begin{tabular}{lllll}
\hline F: Status of the devices & A & M & N.A. & Specific criteria \\
\hline F1: Uniform icons and symbols & --- & --- & --- & Yes $=5$, No $=0$ \\
F2: Status team representativeness & --- & --- & --- & $l \mathrm{le}<4=5$, le $>4=0$ \\
\hline
\end{tabular}

There is no use of symbols therefore the uniform icons and symbols sub-indicator (F1) got a " 0 " and the status team representativeness (F2) got a " 5 ", having " 2.5 " points in total for the status and devices indicator $(\mathrm{F})$.

Table 8. Process values

\begin{tabular}{lllll}
\hline G: Process values & A & M & N.A. & Specific criteria \\
\hline G1: Visibility & 5 & 3 & 0 & --- \\
G2: Location & 5 & 3 & 0 & --- \\
\hline
\end{tabular}

The visibility subindicator (G1) got a " 3 " because not all the required values are visible (for example is not easy to find the altitude value) and in general the process values could be better visualized. The location subindicator (G2) got a "3" because the process values are relatively well located, but it could be easier for the operator. These values lead to " 3 " points in total for the process values indicator $(G)$.

Table 9. Graphs and tables

\begin{tabular}{lllll}
\hline H: Graphs and tables & A & M & N.A. & Specific criteria \\
\hline H1: Format & 5 & 3 & 0 & --- \\
H2: Visibility & 5 & 3 & 0 & --- \\
H3: Location & 5 & 3 & 0 & --- \\
H4: Grouping & 5 & 3 & 0 & --- \\
\hline
\end{tabular}


The format of the graphs and tables is appropriate therefore the subindicator (H1) got a "5", the visibility (H2) and location (H3) subindicators got a "3" because some graphics could be a supplement, like displaying the advanced distance that depends of the speed and the time and the graphs and tables are relatively well located. Since there is not any suitable grouping the subindicator (H4) got a " 0 ", having " 2.75 " points in total for the graphs and tables indicator $(\mathrm{H})$.

Table 10. Data entry commands

\begin{tabular}{lllll}
\hline I: Data entry commands & A & M & N.A. & Specific criteria \\
\hline I1: Visibility & 5 & 3 & 0 & --- \\
I2: Usability & 5 & 3 & 0 & --- \\
I3: Feedback & 5 & 3 & 0 & --- \\
\hline
\end{tabular}

The visibility (I1) got a "3" because the input commands could be better visualized, the usability (I2) got a " 5 " and the feedback (I3) got a " 3 " because the feedback is indirect, the operator must enter a command to know about a new situation, when it should be automatic. These values lead to " 3.67 " points in total for the data entry commands indicator (I).

Table 11. Alarms

\begin{tabular}{lllll}
\hline J: Alarms & A & M & N.A. & Specific criteria \\
\hline J1: Visibility of alarms & 5 & 3 & 0 & -- \\
J2: Location & 5 & 3 & 0 & --- \\
J3: Situation awareness & --- & --- & --- & Yes=5, No=0 \\
J4: Alarms grouping & 5 & 3 & 0 & --- \\
J5: Information to the operator & 5 & 3 & 0 & --- \\
\hline
\end{tabular}

The visibility of alarms ( $\mathrm{J} 1)$ got a " 3 " because the alarms could be better visualized; the location (J2) got a "3" because the alarms are relatively well located. The alarms could give better parameters or instructions to let the operator have a better understanding of the situation, therefore the situation awareness (J3) got a " 3 ". In general the alarms grouping and how the alarms are showed to the operator are correct therefore the subindicators (J4) and (J5) got a " 5 ". All these values lead to "3.8" points in total for the alarms indicator $(J)$. The final result of the Sky-eye graphic user interface evaluation, taking into account that each indicator has the same weight, stands in 2.572 (rounded = 2.6). After applying some corrections to the detected anomalies, the global evaluation index can be stood between 4 and 5 which are the maximum values of the numeric scale. 


\section{Proposals for Interface Improvements}

The GEDIS-UAV analysis and evaluation made possible to identify an interface limited in structure, distribution and navigation. These three first indicators show the errors that occur more often in the design of GUIs for UAVs.

Among other things it was detected that the text size is not right, the interface does not show the status of the devices in a suitable manner, the process values could be better visualized as well as the data-entry commands; all this increases the operator response time and the delays inside the control loop. Another important anomaly detected is that the interface's alarms could give more information in case of failures in order to improve the operator situational awareness [6]. Figure 2 depicts the distribution analysis of the Sky-eye project GUI. Figure 2A depicts the original GUI distribution and figure $2 \mathrm{~B}$ shows the proposed distribution.

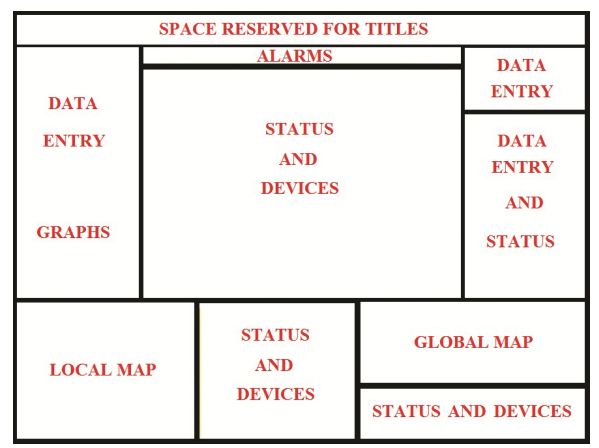

(A) Original GUI distribution

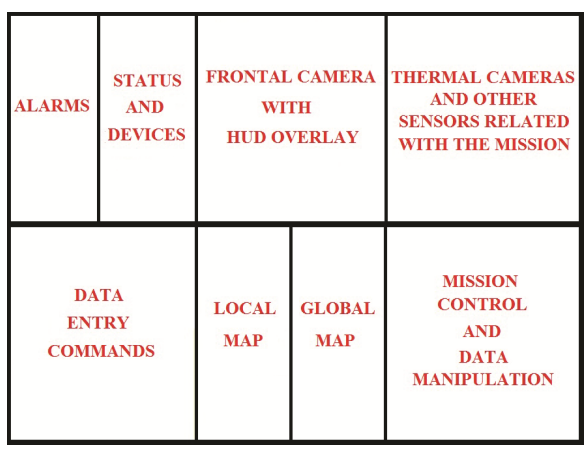

(B) Proposed distribution

Fig. 2. Distribution analysis of the Sky-eye project GUI

As part of the recommendations the following screens were proposed: Data entry commands, alarms, UAV systems status and process values, local map, global map, frontal video with HUD (head-up display) overlay, thermal cameras and mission control/ data manipulation (shown in figure 3). Each screen represents one of the zones that the interface is supposed to have, and should meet specific ergonomic criteria.

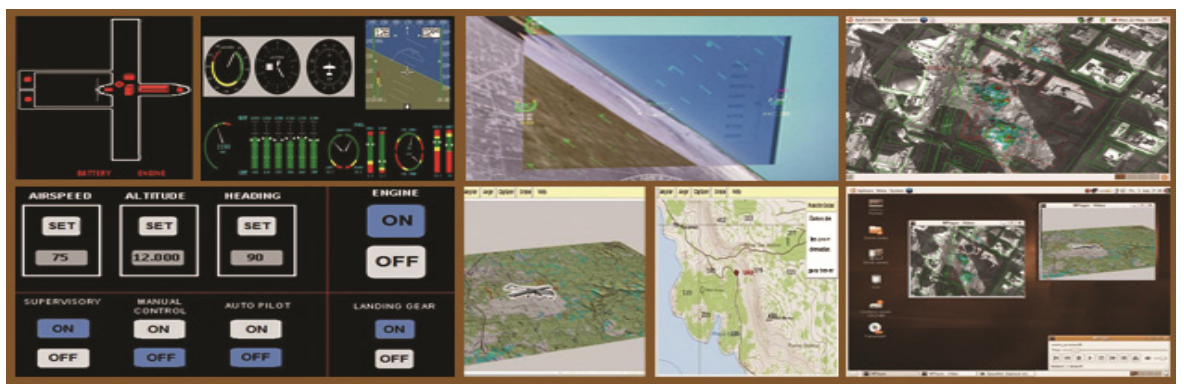

Fig. 3. Proposed GUI for Sky-eye two-wing UAV 
At a glance is possible to note the difference and the level of improvement. The evaluation of the proposed interface through GEDIS-UAV guide is 5, which is the maximum value of the numerical scale; this means that the implementation of the GUI will minimize at maximum the possibility of human error.

\section{Conclusions and Future Work}

As a result and based on GEDIS-UAV recommendations, the students of the BarcelonaTech improved the interface design for the "Shadow MK1" UAV [10], [17]. For more details about the work conducted in order to design the graphical user interface refer to "Diseño de Interfaces de Supervisión de Vehículos Aéreos No Tripulados Supervisory Control Interfaces Design for UAVs" [9].

Although there are some standards regarding security for human machine interface systems that keep relation with physical ergonomics and interface design aspects through style rules, it is remarkable the absence of human centered designs in interactive systems. At this point, our contribution is to apply usability engineering, using techniques like the measurement of the operator mental workload, improvement of the GUI design and others related with usability techniques like the cognitive walkthrough. The application of GEDIS-UAV guide on a real project, demonstrates the functionality and applicability of the guideline. Future work will try to establish more specific ergonomic criteria to design supervisory interfaces by adding, deleting or modifying some indicators. Another line of research will try to improve the guide assessment techniques, in the present work, the evaluation methodology assigns the same weight to all the indicators, but maybe this could be improved if it is taken into account the importance of some indicators over others. Important efforts of future research will take into account the use of new input devices like multi-touch screens, speech recognition engines and brain wave sensors in order to improve the design and implementation process of interfaces for unmanned vehicles.

\section{References}

1. Air Force Research Laboratory. Wright-Patterson Air Force Base (October 2007), http://www.wpafb.af.mil/shared/media/document/ AFD-070418-024.pdf

2. ARINC: Aeronautical Radio. Incorporated (2010), http: / / www .aviation-ia.com/aeec/projects / cds / index.html

3. Carrigan, G.P., Long, D., Cummings, M.L., Duffner, J.: Human Factors Analysis of Predator B Crash. In: Proceedings of AUVSI 2008: Unmanned Systems North America, San Diego, California (June 2008)

4. Cummings, M.L., Kirschbaum, A.R., Sulmistras, A., Platts, J.T.: STANG 4586 Human Supervisory Control Implications. In: Proceedings of UVS Canada Annual Conference, Montebello, Quebec, Canada (2006)

5. Cooper, J.G.: Cognitive Engineering Research Institute (June 2007), http: / / www.cerici.com/documents / 2007_workshop/ 6-1-BYU-Cooper.pdf 
6. Endsley, M.: Measurement of situation awareness in dynamic systems. Human Factors (1995)

7. Foundation, F. S. Flight Safety Digest, Alexandria, Virginia, United States (May 2005), http://flightsafety.org/fsd/fsd_may05.pdf

8. ISO: International Organization for Standardization (2000),

http://www.iso.org/iso/iso_catalogue/

catalogue_tc/catalogue_detail.htm?csnumber $=30030$

9. Lorite, S.: Diseño de interfaces de supervisión de vehículos aéreos no tripulados (2008), http://www. institutoidf.com/instituto/downloads/

user_center_design_interfaces/

Interfaces_Vehiculos_Aereos_No_Tripulados.pdf

10. Martinez, J.: AutoNAV4D. A co-simulator for Unmanned Aircraft Systems. BarcelonaTech, Castelldefels (2008)

11. NATO: The North Atlantic Treaty Organization (2007),

http: / / www . nato. int/structur/AC/224/standard/AEDP2/

AEDP2_Documents / AEDP-02v1.pdf

12. Nimmo: Designing control rooms for humans. Control Magazine (2004)

13. Owen, D.: Air Accident Investigation, New Edition. PSL Patrick Stephens Limited, Somerset England (2001)

14. Pastor, E., Royo, P., Lopez, J., Barrado, C., Santamaria, E., Prats: Project SKY-EYE. Applying UAVs to Forest Fire Fighter Support and Monitoring. In: UAV 2007 Conference (2007)

15. Pastor, E., Lopez, J., Royo, P.: A Hardware/Software Architecture for UAV Payload and Mission Control. In: 25th Digital Avionics Systems Conference (2006)

16. Pastor, E., Lopez, J., Royo, P.: An Embedded Architecture for Mission Control of Unmanned Aerial Vehicles. In: 9th EuroMicro Conference on Digital Systems (2006)

17. Pastor, E., Prats, X., Royo, P., Delgado, L., Santamaria, E.: UAS Pilot Support for Departure, Approach and Airfield Operations. In: Proceedings of the IEEE Aerospace Conference, Montana, United States (2010)

18. Ponsa, P., Díaz, M.: Creation of an Ergonomic Guideline for Supervisory Control Interface Design. In: Harris, D. (ed.) HCII 2007 and EPCE 2007. LNCS (LNAI), vol. 4562, pp. 137-146. Springer, Heidelberg (2007)

19. Ponsa, P., Vilanova, R., Amante, B.: Human intervention and interface design in automation systems. International Journal of Computers, Communications \& Control 6(1), 166$174(2011)$

20. RTCA: Radio Technical Commission for Aeronautics, and the European Organization for Civil Aviation Equipment EUROCAE (1992), http: / /www. do178site.com/

21. Schneiderman, B.: Designing the user interface. Strategies for effective human-computer interaction, 3rd edn. Addison-Wesley (1998)

22. Sheridan, T.B.: Telerrobotics, automation and human supervisory control. M.I.T. Press (1992)

23. Society of Automotive Engineers SAE, Unmanned Systems Technical Committee AS4 (2010), http: / / standards.sae.org/as5684a/

24. Strauch, B.: Investigating Human Error: Incidents, Accidents and Complex Systems. Ashgate Publishing (2002)

25. Terrence Fong, C.T.: Vehicle Teleoperation Interfaces. Autonomous Robots 11, 9-18 (2001)

26. William, T.: U.S. Military Unmanned Aerial Vehicle Mishaps: Assessment of the Role of Human Factors Using Human Factors Analysis and Classification System (HFACS) (2005), http: / /www .wpafb.af .mil/shared/media/document/

AFD-090226-154.pdf 